\title{
Dimensional Reduction of Data for Anomaly Detection and Speed Performance using PCA and DBSCAN
}

\author{
A. Yugandhar Srihari, Sashirekha. K
}

\begin{abstract}
Anomaly detection is the major problem facing by many of industries. It includes network intrusion and medical sciences. Several fields like Astronomy and research also facing difficulties in finding effective anomaly detection. They have included several techniques to solve such problems. Clustering is the technique which has been employed by many of the researchers. The most commonly used algorithm to perform clustering is DBSCAN. It is well known clustering algorithm used in data mining and Machine learning. It is referred as Density based spatial clustering of application with noise. Because of its high complexity in computation, it must be decreased in terms of dimensionality of data points. PCA is a method used then to reduce dimensionality and produced a new data set which is again undergo DBSCAN. Here by the nature of the test results was precise there by such a methodology can be adjusted. The mix of PCA and DBSCAN was acutely confirmed and resultant examination shows that a speedup of $25 \%$ was improved while the quality was $80 \%$ diminishing the dimensionality of informational index of half.
\end{abstract}

Keywords: Anomaly detection, DBSCAN, PCA.

\section{INTRODUCTION}

Data mining technologies used to process large amount of data to retrieve desired content. A large amount of data consists of both structured and unstructured data which is to be analysed and processed using several classification algorithms. In simple terms data mining is technique of mining knowledge from given big volume of data. The major obstacle in processing data is detecting anomalies. Usually anomalies are unexpected results or deviated results rather than norm. Data clustering is a technique used for effective identification of points in the data sets which can be termed as outliers or noise. The most popular technique in clustering of data is DBSCAN. Based on Euclidean distance measurement the points which are closed together are grouped using DBSCAN. It also marks as outliers the points that are in low-density regions.

DBSCAN algorithm basically requires two parameters as input. It is used to specify how much close the points should be to each other to be considered a part of a cluster. It is viewed as neighbours if the separation between two is lower or equivalent to epse steem. Min Points are to discover least number of focuses to shape a thick area.

Revised Manuscript Received on December 12, 2019

A. Yugandhar Srihari, Saveetha School of Engineering Saveetha Institute of Medical and Technical Sciences Chennai, India

Sashirekha. K, Associate Professor, Saveetha School of Engineering, Saveetha Institute of Medical and Technical Sciences Chennai, India

For instance, in the event that we set the min Points parameter as 5 , at that point we need at any rate 5 points to shape a thick district. There are several methods used for dimensionality reductions such as PCA, LDA, and Generalized Discriminant Analysis. Dimensionality reduction is of both linear and nonlinear. PCA is considered to be prime component analysis. This method was first introduced by Karl Pearson. It works on higher dimensional data is mapped to the data of lower dimensional data. It helps in data compression and reduces computation time. It also helps in redundant values.

\section{LITERATURE SURVEY}

Precise dimensionality decrease considers the advancement of quantum look and transport issues on specific charts. Previously, the Lanczos Algorithm is used to reduce dimensionality over network of charts. It includes Complete Graph; It also uses Complete Multipartite Graphs and $\mathrm{CBG}$. We centre around growing the extent of these decreases to the CBG with evenness broken so as to permit the improvement of Quantum Walks on this sort of graph. We show that in like manner to the CG, the Lanczos Algorithm can be reached out to the CBG with broken parity, which has $\mathrm{k}$ irregular edges expelled with the imperatives that close to one edge for each hub is evacuated and that no edges that interface with the arrangement hub are expelled. In contrast to the $\mathrm{CG}$ with broken edges, which, after decrease, has 3 kinds of hubs and a subsequent $3 \times 3$ lattice, the CBG with broken edges diminishes to a chart with 5 sorts of hubs, bringing about a decrease from aNxN framework to a $5 \times 5$ network. From these outcomes, it might be additionally investigated whether the more broad CMPG decrease may likewise be extended by breaking the chart's balance, and assuming this is the case, how the components of the diminished grids will be influenced as the quantity of allotments develops.

Land cover mapping utilizing high pixels picture time arrangement faces the issue managing high volumes of information which can undermine the capacity of regulated classifiers to learn appropriate choice limits. In spite of the fact that dimensionality decrease approaches have been applied to hyper spectral symbolism for quite a while, their utilization with thick time arrangement has not yet been investigated. We study the handiness of dimensionality decrease as a pre-handling step for high goals optical picture time arrangement managed characterization for land spread mapping. 
Head Component Analysis (PCA), Auto encoders and Kohonen's Self Organizing Map are looked at more than 3 dimensionality decrease draws near: worldwide, per date and per band. Applying PCA to each date of the time arrangement yields the best outcomes as far as characterization exactness.

Hyper spectral information with high dimensionality in every case needs more storage leads to increase in computational utilization, complex learning is widely utilized in dimensionality decrease. A tale dimensionality decrease technique dependent on complex learning is proposed through learning a steady nearby complex portrayal. Four contiguousness charts are built to display the interclass likeness, interclass decent variety, intra class similitude and intra class assorted variety individually, and afterward combine these diagrams into the discriminant target work for direct dimensionality decrease. The order brings about the utilization of various techniques are contrasted and exploratory outcomes show that the proposed strategy is compelling and it is better than examination strategies.

PCA and LDA are the most outstanding strategies to diminish the dimensionality of featured vectors.LDA is referred as Linear Discriminant Analysis .The two strategies face difficulties when utilized on multi label information every data point might be related to different labels. PCA doesn't exploit the label data in this way the performance is yielded. LDA can misuse class data for multiclass information, yet can't be straightforwardly applied to multi label issues. In this context, we propose a dimensionality decrease strategy for multi label information. We initially present the summed up Hamming separation that estimates the separation of two information focuses in the mark space. At that point the proposed separation is utilized in the diagram installing system for include measurement decrease. We confirmed the proposed strategy utilizing three multi label benchmark datasets and one enormous picture dataset. The outcomes show that the proposed highlight dimensionality decrease strategy reliably outflanks PCA and other contending methods.

\section{RELATED WORK}

In detail there are two types of anomaly detection 1) making the outliers 2) verification of anomaly probability. Our context is based on first category hence we neglect the second category. The detailed explanation of how we integrated and used both DBSCAN and PCA are as follows

A. DBSCAN Identification of neighbouring points, compare according to min points to create large cluster. Resultant consists of list of clusters and outliers.

B. Dimensionality reduction is the main agenda of PCA

1) Formation of matrix $N X D(N$ is referred as amount of data and $\mathrm{D}$ is referred as Dimension)

2) Evaluate mean vector of D- Dimension

3) Calculate the co-variance

Formation of new data set.

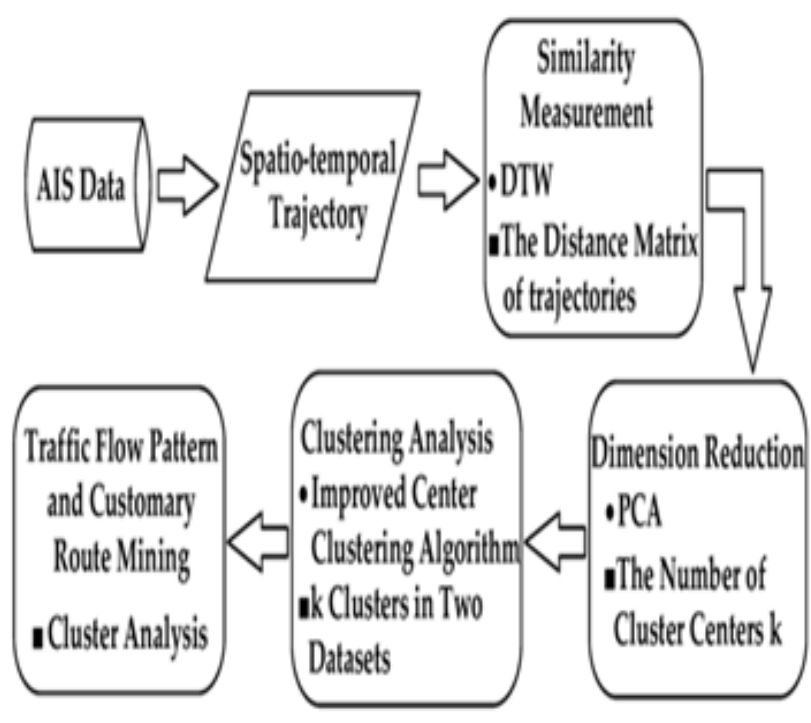

\section{Architecture of Dimensional Reduction}

\section{EXPERIMENTAL RESULTS}

To do experimental work we need to have minimum of INTEL(R)CORE (TM) i5-5200U CPU with $2.20 \mathrm{GHz}$ processor and 8.00 GB RAM with ubuntu 16.04 LTS. operating system, and gcc version 5.4.0.

Table. 1 PCA QUALITY (1 DAY)

\begin{tabular}{|c|c|}
\hline $\begin{array}{c}\text { Dimension of data } \\
\text { points }\end{array}$ & Quality \\
\hline 8 & $100.00 \%$ \\
\hline 7 & $99.64 \%$ \\
\hline 6 & $99.17 \%$ \\
\hline 5 & $98.23 \%$ \\
\hline 4 & $94.83 \%$ \\
\hline 3 & $83.82 \%$ \\
\hline 2 & $71.48 \%$ \\
\hline 1 & $58.69 \%$ \\
\hline
\end{tabular}

Table 2 represents quality of PCA after reducing dimension

Table. 2 PCA QUALITY(1 MONTH)

\begin{tabular}{|c|c|}
\hline $\begin{array}{c}\text { Dimension of data } \\
\text { points }\end{array}$ & Quality \\
\hline 8 & $100.00 \%$ \\
\hline 7 & $97.48 \%$ \\
\hline 6 & $93.84 \%$ \\
\hline 5 & $88.94 \%$ \\
\hline 4 & $80.71 \%$ \\
\hline 3 & $71.07 \%$ \\
\hline 2 & $60.08 \%$ \\
\hline 1 & $37.32 \%$ \\
\hline
\end{tabular}


Table. 3 Performance of DBSCN AND PCA vs DBSCAN $(\operatorname{dim}=8)$

\begin{tabular}{|c|c|c|c|c|}
\hline $\begin{array}{c}\text { Di } \\
\mathrm{m}\end{array}$ & DBSCAN & PCA & $\begin{array}{c}\text { DBSCAN } \\
\text { N-8 dim }\end{array}$ & $\begin{array}{c}\text { DBSCAN+PC } \\
\mathrm{A}\end{array}$ \\
\hline 8 & 4.47 & 0.00 & 4.47 & 4.47 \\
\hline 7 & 4.12 & 0.43 & 4.47 & 4.55 \\
\hline 6 & 3.90 & 0.42 & 4.47 & 4.32 \\
\hline 5 & 3.63 & 0.38 & 4.47 & 4.01 \\
\hline 4 & 3.35 & 0.36 & 4.47 & 3.71 \\
\hline 3 & 3.21 & 0.31 & 4.47 & 3.51 \\
\hline 2 & 3.14 & 0.27 & 4.47 & 3.41 \\
\hline 1 & 2.62 & 0.25 & 4.47 & 2.87 \\
\hline
\end{tabular}

The exploratory outcomes got from this work demonstrate that the mix of PCA and DBSCAN can create after effects of high calibre while expanding huge the execution. Our examination indicated that in all cases, when the nature of DBSCAN is diminishing, the created point irregularities are equivalent to when $\operatorname{dim}=8$ however not every one of them. This implies there are not various yields but rather less point irregularities. Here, it is a decent question to look at if these focuses are of higher hazard than the others (delivered when $\mathrm{D}=8$ ). Another significant issue is that for all the created results the first DBSCAN was utilized. There are a ton of parallel/circulated varieties of this calculation which could increment further its presentation, In the opposite, for PCA a multi-center form was utilized (OpenMP) parallelizing all circles when it was conceivable.

\section{CONCLUSION}

To reduce the dimensionality proposed method consists combination of PCA and DBSCAN for a given input dataset. The resultant we get are very promising and conceptually verified. Upcoming work will focus on performance includes techniques using distributed, parallel and multi-core GPU.

\section{REFERENCES}

1. V. Chandola, A. Banerjee, V. Kumar, "Anomaly Detection for Discrete Sequences: A Survey”, In: IEEE Transactions on Knowledge and Data Engineering, vol. 24, no. 5, 2012. pp. 823-839.

2. M. Ester, H. Kriegel, J. Sander, and X. Xu., "A density based algorithm for discovering clusters in large spatial databases with noise", In: KDD-96 Proceedings, pp. 226-231,

3. J. Gan and Y. Tao, "Db scan revisited: Mis-claim, un-fixability, and approximation", In Proceedings of the 2015 ACM SIGMOD International Conference on Management of Data, SIGMOD '15,pages 519-530, New York, NY, USA, 2015. ACM

4. K. Pearson, "On Lines and Planes of Closest Fit to Systems of Points in Space", Philosophical Magazine, 2 (11), pp. 559-572.

5. O. I. Sheluhin, S. M. Smolskiy, A. V. Osin, "Self-Similar Processes in Telecommunications". John Wiley \& Sons, Ltd, 2007.316 p.

6. J. Beran, Y. Feng, S. Ghosh, R. Kulik, "Long-Memory Processes. Probabilistic Properties and Statistical Methods". Springer, Berlin, Heidelberg, 2013. 884 p.

7. M. E. Crovella, M.S. Taqqu, and A. Bestavros, "Heavy-tailed probability distributions in the World Wide Web". In: A Practical Guide to Heavy Tails: Statistical Techniques and Applications, R. J. Adler, R.E. Feldman, and M.S. Taqqu (Eds.), Birkhäuser, Boston/1998. pp. 3-25.

8. I. Syarif, A. Prugel-Benett, and G. Wills, "Unsupervised Clustering Approach for Network Anomaly Detection", In: Benlamri R.
(eds)Networked Digital Technologies. NDT 2012. Communications in Computer and Information Science, vol 293. Springer, Berlin, Heidelberg.

9. A. V. Chernov, I. K. Savvas, and M. A. Butakova, "Detection of Point Anomalies in Railway Intelligent Control System Using Fast Clustering Techniques", 3rd International Scientific Conference "Intelligent Information Technologies for Industry, 2018, Springer.

10. S. Thiprungsri, and M. A. Vasarhelyi, "Cluster Analysis for Anomaly Detection in Accounting Data: An Audit Approach", The Int. Journal of Digital Accounting Research, vol.11, 2011, pp. 69-84. 